\title{
Process of desalination of low energy consumption and high compatibility with the use of renewable energies
}

\author{
Pedro Peñas Ballester ${ }^{1}$, Francisco Javier Abad Garrido ${ }^{1}$ \\ ${ }^{1}$ Electrical Engineer \\ Phone/Fax number:+0034 652880706, e-mail: pedro.penasb@ieee.org, fj.abad.garrido@gmail.com
}

\begin{abstract}
This document shows the theoretical justification of a new process of desalination with lower energy consumption than reverse osmosis and with a high compatibility for its use with the renewable energies.
\end{abstract}

The new desalination process involves the use of electrodialysis concepts regarding the use of charged- ion selective membranes to create differentiated cells of concentrated salt water and diluted or desalinized water. However, the concept of transporting ions changes; in this case, with respect to electrodialysis, since in order to transport the ions, it is used the second term of Lorentz equation instead the first term. In the first term, there is an electric field, which is used by conventional electrodialysis; whereas, in the second term, there is the joint action of a magnetic field together with the velocity of the fluid to cause the motion of electric charges according to their signs. The use of a magnetic field instead of the use of an electric field can be an advantage when trying to solve the electro-neutrality of the cells of the edges without having to turn to the use of redox reactions.

This process of desalination works from two main parameters.

- One permanent magnetic dipole. Therefore the energy of this magnetic field remains inalterable throughout the time.

- The electric charge speed, on the desalted water, versus the magnetic field of the magnetic dipole. This speed does not have to be high.

Key words. Desalination, magnetic, field, electrohydrodinamic, membranes.

\section{Introduction}

One of the great problems in the sea water desalination is the high power consumption by cubic metre of produced water. The best alternative to date has been the desalination by reverse osmosis. Despite this process he is not compatible with the use of renewable energies.
The seawater desalination technology is at a standstill, at specific consumption limits that are above $300 \%$ of the necessary theoretical minimum value. The main cause of these consumptions is the behaviour of the membranes of reverse osmosis. The specific consumption of reverse osmosis is the lowest of existing technologies. Nevertheless, there is still much more to improve if we want to obtain a desalination process in order to approach the notional specific minimum consumption prescribed by the laws of thermodynamics.

The desalination process must lower its cost and specific consumption of electrical energy if the aim is to qualitatively trigger the supply of potable water, but above all, the supply of irrigation water. For this reason, during the last years, new lines of investigation have been followed to try to find those development lines that are capable of opening desalination roads where thermodynamic irreversibilities derived from used technology are minimal. One of the lines investigated and theoretically compared to offers successful expectations to achieve the desired objective. The following project is presented in order to develop this line of investigation.

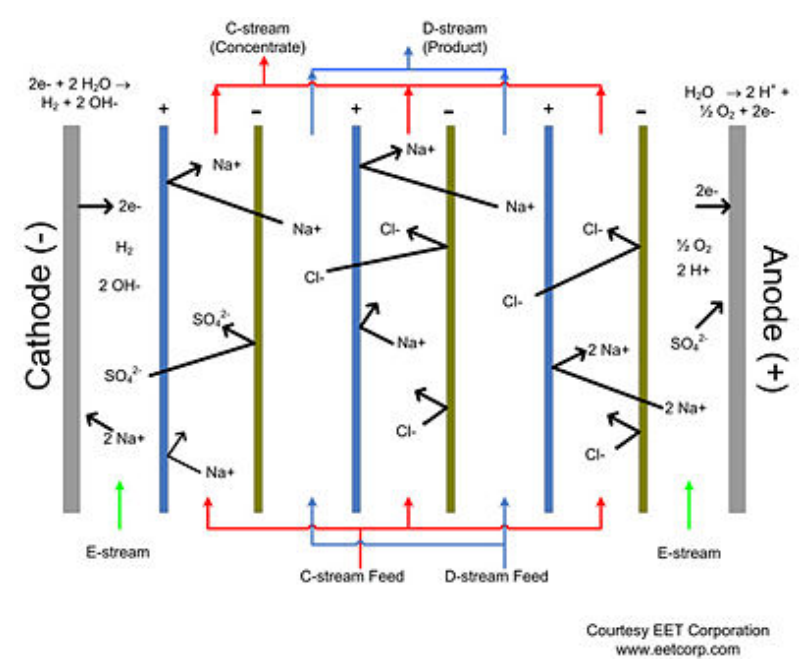

Fig. 1. Stack of electrodyalisis cells 


\section{Base Technologies for the present desalination Project (E+MHD)}

The new desalination process involves the use of electrodialysis concepts regarding the use of charged- ion selective membranes to create differentiated cells of concentrated salt water and diluted or desalinized water. However, the concept of transporting ions changes; in this case, with respect to electrodialysis, since in order to transport the ions, it is used the second term of Lorentz equation instead the first term. In the first term, there is an electric field, which is used by conventional electrodialysis; whereas, in the second term, there is the joint action of a magnetic field together with the velocity of the fluid to cause the motion of electric charges according to their signs.

The electrodialysis is a process of desalination. This process of desalination consists of the ion exchange of salt water between separated channels by selective membranes (Fig. 1).

The principle of operation of the electrodialysis represents the Lorentz force.

In physics, the Lorentz force is the force on a point charge due to electromagnetic fields. It is given by the following equation in terms of the electric and magnetic fields:[1]

$$
\mathbf{F}=q[\mathbf{E}+(\mathbf{v} \times \mathbf{B})],
$$

Where:

$\boldsymbol{F}$ is the force (in newtons)

$\boldsymbol{E}$ is the electric field (in volts per meter)

$\boldsymbol{B}$ is the magnetic field (in teslas)

$q$ is the electric charge of the particle (in coulombs)

$v$ is the instantaneous velocity of the particle (in meters per second)

$x$ is the vector cross product

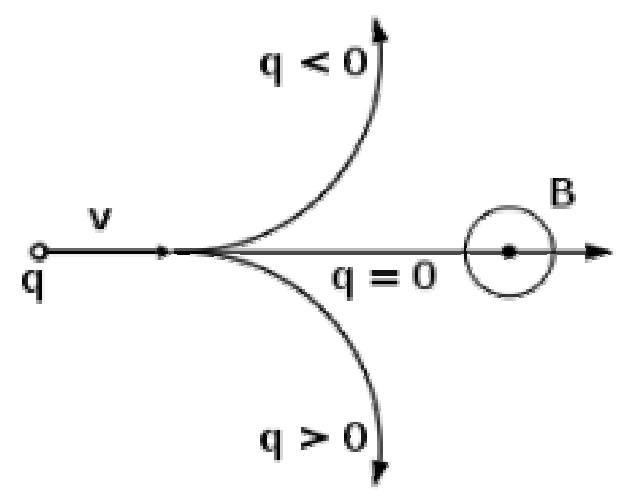

Fig. 2. Trajectory of a particle with charge q, under the influence of a magnetic field B directed perpendicularly out of the paper, for different values of q.

In the electrodialysis the movement of ions by the force takes place that exerts the electric field, in electrodialysis
B is equal zero, according to the Lorentz force, on the ionic charge of salt water.

The electrodialysis has a series of problems that cause that it has high power consumption. These problems can be summarised in the following points.

- The electric field is transmitted of linear form.

- The stack of cells of electrodialysis has to have a linear dimension.

- This linearity forces makes that at least two cells, in the ends, have unbalanced ionic charges.

- In sensible cells one internal electric field are created due to unbalanced electrics charges. This internal electric field neutralise the outer electric field and therefore stops the desalination.

- To prevent that the desalination is stopped is necessary to balance the electric charges in the cells of the ends.

- This balance is realised with oxidation reactions in a cell and reduction in the other cell.

- These reactions of oxidation and reduction require the use of an intensity of electrons by the circuit.

- This electrical intensity is the responsible of most of the electrical losses by Joule effect. Mainly these losses must to the electrical high resistance of the diluate cells.

This problem results from the need to maintain the electro-neutrality in the cells of the edges. Whereas, the last problem is the consequence of the difference of concentrations in the channels. The use of a magnetic field instead of the use of an electric field can be an advantage when trying to solve the electro-neutrality of the cells of the edges without having to turn to the use of redox reactions.

There is a constructive form (Fig. 3) to prevent that it is necessary the use of an intensity of electrons. This constructive form consists of the use of a geometrical configuration that does not have cells in the ends.

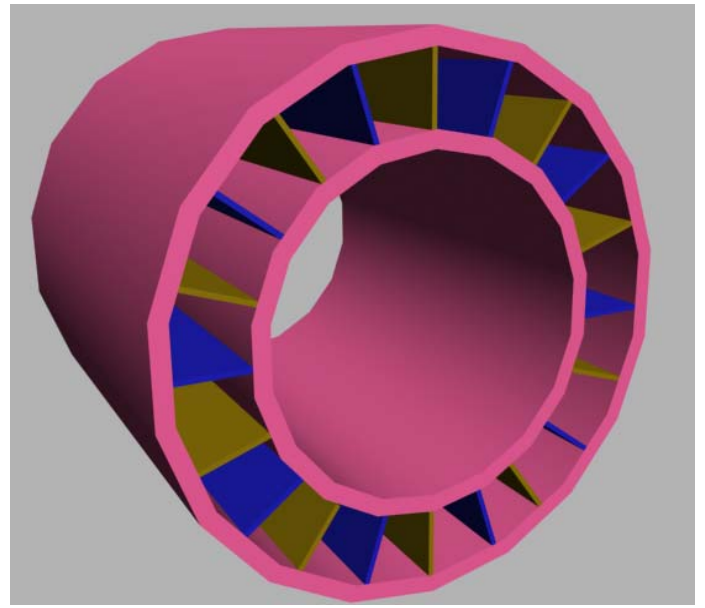

Fig. 3. Stack of MHD desalination cells

This configuration is not compatible with a force due to an electric field. We can not use de electric field term of the Lorentz force but we can use the second term of the 
Lorentz force. This term is developed in called science Magnetohydrodynamics, (magnetofluiddynamics or hydromagnetics) that is the academic discipline which studies the dynamics of electrically conducting fluids. Examples of such fluids include plasmas, liquid metals, and salt water.

Having said that, there are some advantages of this process in relation to the electrodialysis process that should be pointed out. Among them:

- No source of electrical energy in the form of continuous current is used to carry out the electrodialysis.

- The necessary energy for the process is directly communicated to the ion by the velocity of the fluid thanks to a magnetic coupling. The greater the existing magnetic field is, the greater the efficiency of this transmission of energy.

- No Electric resistance in the diluate channels.

- No Consumed energy due to redox reactions.

However, there are still the same problems that with electrodialysis:

- Growth of the Donnan and Nernst potentials due to the increasing difference of concentration between the diluate channel and the concentrated channel.

The only irreversibilities that are left are the ones derived from the Donnan and Nernst potentials but such can be minimized leading to a desalination system by phases.

Thanks to the different possibilities from configuration of a magnetic dipole it is possible to draw a geometrical configuration of cells formed by selective membranes to cations and anions that are become ordained of alternate form.

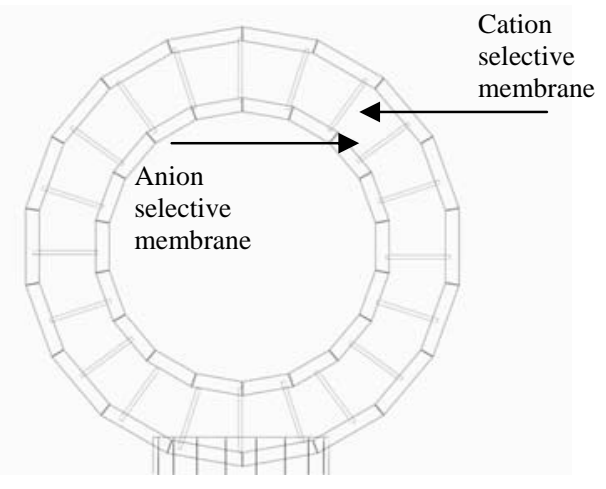

Fig. 4. Stack of MHD desalination cells

With this geometrical configuration and a suitable magnetic field is possible to carry out the desalination of the sea water with the electrodialysis membranes without the same energetic losses.

The power consumption of this new process of desalination can be modelized with the Nernst's equation $\{2\}$ together MHD equation $\{3\}$ and the Lorentz force equation $\{1\}$.

$$
\begin{aligned}
& V=V_{1}-V_{2}= \pm 2,3 \frac{k T}{e} \log \frac{c_{1}}{c_{2}} \\
& \nabla P=\mathbf{j} \times \mathbf{B}+\eta \nabla^{2} \mathbf{v},
\end{aligned}
$$

This process of desalination works from two main parameters.

- $\quad$ One permanent magnetic dipole (Fig. 5). Therefore the energy of this magnetic field remains inalterable throughout the time.

- The electric charge speed, on the desalted water, versus the magnetic field of the magnetic dipole. This speed does not have to be high.

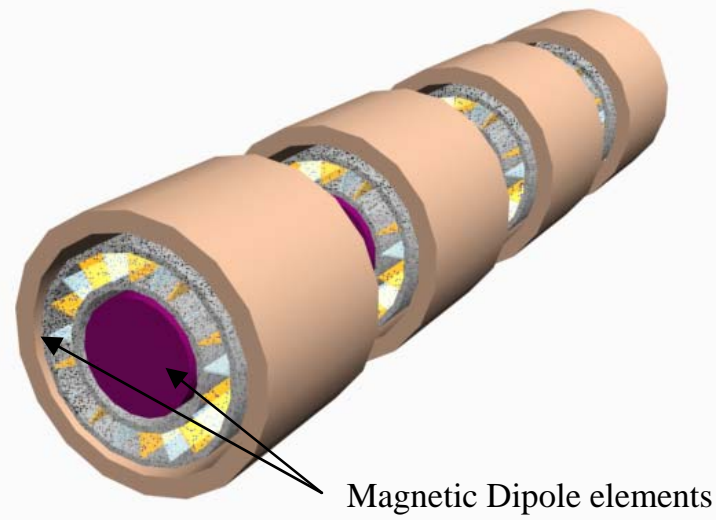

Fig. 5. Module of stacks of MHD desalination cells

\section{A. Magnetic field.}

One of the main technological goals of this process of desalination is the use of strong magnetic fields. The magnetic fields must be of the order of 5 Tesla. In order to get this magnetic field we may use superconductivity dipole.

The Ciemat, Research centre Energetics, Environmental and Technological of Spain, already has realised predesigns for the construction of one magnetic dipole adapted to this process of desalination of sea water.

\section{B. Compatibility with the use of renewable energies}

The actually desalinisations methods needs a constant flow of energy. In this case we have two ways to get the adaptation between renewable energy and power energy need in de desalinisation process.

The firs way is to produce and accumulate filter sea water inside of one tank when the power renewable energy is high. When the renewable energy is low we can use de water accumulated in the tank.

The second way is modify the total flow water produced. This way is possible when we have a modular system. This modular system can be connected in serial or in 
parallel form in order to get the necessary adjust between the Lorentz Force and the Nernst potential.

\section{Estimation of the specific consumption of the E+MHD}

Following are the specific consumption for E+MHD and reverse osmosis process.

TABLE I. - Specific consumption

\begin{tabular}{|r|r|r|r|}
\hline \multicolumn{1}{|c|}{$\begin{array}{c}\text { Fonversion } \\
\text { Factor }\end{array}$} & $\begin{array}{c}\text { E+MHD } \\
\text { ideal } \\
(\mathrm{kwh} / \mathrm{m} 3)\end{array}$ & $\begin{array}{c}\text { R.O. } \\
\mathrm{kwh} / \mathrm{m} 3 \\
\text { ideal [5] }\end{array}$ & $\begin{array}{c}\text { R. O. } \\
\mathrm{kwh} / \mathrm{m} 3 \text { real } \\
{[5]}\end{array}$ \\
\hline 0,1 & 0,458 & & \\
\hline 0,2 & 0,486 & & 2,9669 \\
\hline 0,3 & 0,519 & 0,9750 & 2,7998 \\
\hline 0,4 & 0,558 & 1,0516 & 2,8927 \\
\hline 0,5 & 0,607 & 1,1477 & 3,3284 \\
\hline 0,6 & 0,671 & 1,2742 & \\
\hline 0,7 & 0,758 & & \\
\hline 0,8 & 0,889 & & \\
\hline 0,9 & 1,136 & & \\
\hline
\end{tabular}

\section{References}

\section{Acknowledgement}

- Juan Manuel Ortiz Guerra. (Dto. Electroquímica. Universidad Alicante.

- Luis Garcia Tabarés. (Director of superconductivity laboratory CIEMAT in Madrid, Spain).

[1] N.S. Demidova, "Electromagnetic stimulation of electric mass transfer in the flow electrochemical system”, Russian journal of electrochemistry, 1998, vol. 34, nº12, pp. 1265-1273

[2] J. Veerman, J.W. Post, M. Saakes, S.J. Metza, G.J. Harmsenb Wetsus, "Reducing power losses caused by ionic shortcut currents in reverse electrodialysis stacks by a validated model”, Journal of Membrane Science 310 (2008), pp. 418-430

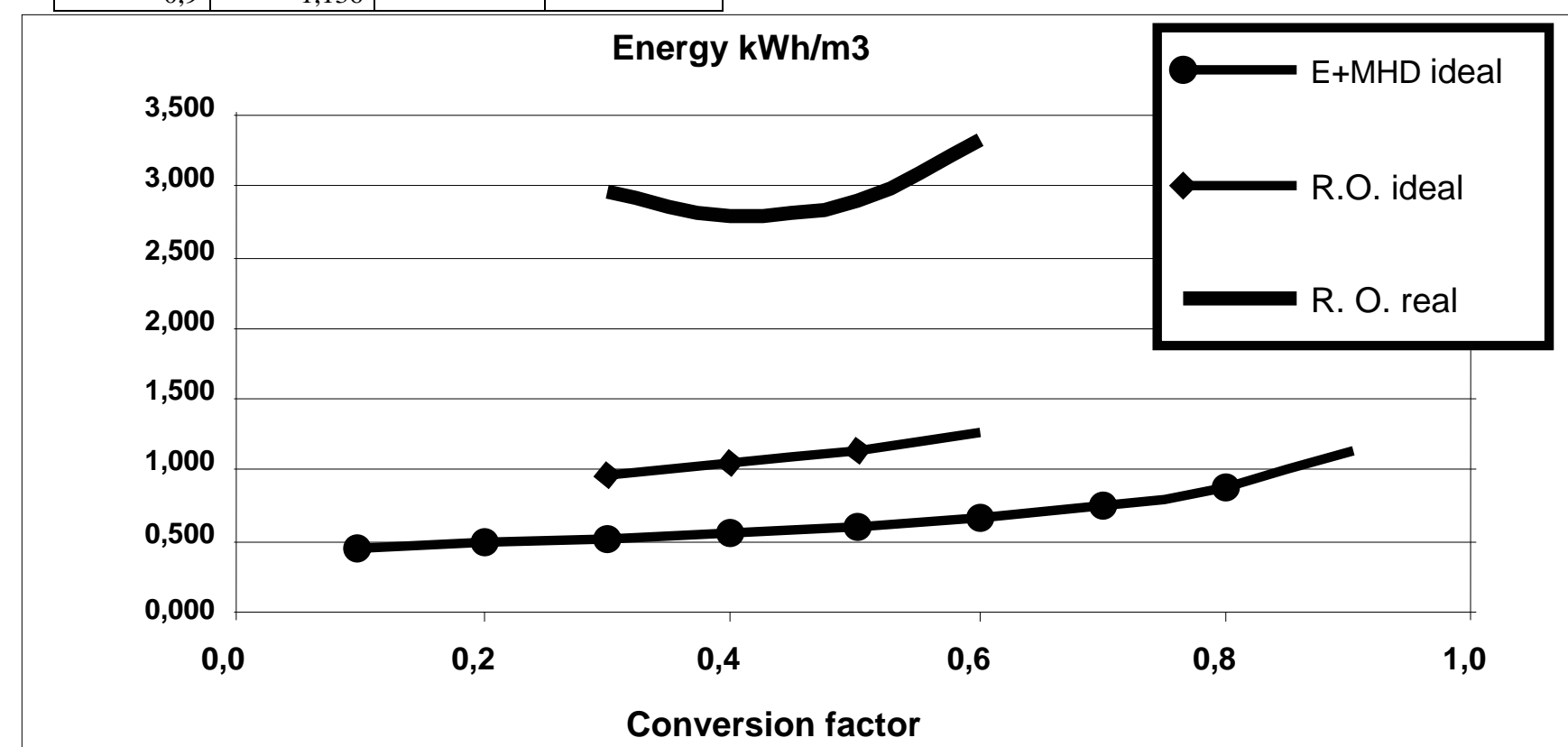

Fig. 6. Graphic comparison of specific consumption

\section{Energetics losses in the magnetic field}

The energetics losses in the magnetic field are due to the loss of heat exclusively. The heat is lost by the walls of the isolation. According to the CIEMAT the energetics losses are very small. It is possible to obtain that the energetics losses are of the order of watts.

\section{Conclusion}

- A new desalinisation concept has been presented.

- This system has theoretical good efficiency consumption.

- This consumption will have, probably, minor than reverse osmosis energy consumption when the real model will be probe.
[3] Minoru Takeda, Naotaka Tomomori, Teruhiko Akazawa, Kazu Nishigaki, and Akira Iwata, "Flow Control of Seawater With a Diverging Duct by MHD Separation Method”, IEEE TRANSACTIONS ON APPLIED SUPERCONDUCTIVITY, VOL. 14, NO. 2, JUNE 2004, pp. 1543-1546

[4] Minoru Takeda, Yasuaki Okuji, Teruhiko Akazawa, Xiaojun Liu, and Tsukasa Kiyoshi, "Fundamental Studies of Helical-Type Seawater MHD Generation System”, IEEE TRANSACTIONS ON APPLIED SUPERCONDUCTIVITY, VOL. 15, NO. 2, JUNE 2005, pp. 2170-2173

[5] Juan María Sánchez, Ruth Sánchez Castillo, "Representación y comparación entre las curvas de operación de un modelo de Planta Desaladora que utiliza como sistema de recuperación de energía SIP y Turbinas Pelton”, VI Congreso Nacional AEDYR, NOVEMBER 2006 